\title{
Linking Protestant Work Ethic to Workplace Ostracism: The Mediating Effect of Subjective Well-Being
}

\section{Suchuan Zhang, Qiao Shi}

School of Economics and Business, Shanxi University, Taiyuan, China

Email address:

zhangsuchuan@126.com (Suchuan Zhang), 18835122426@163.com (Qiao Shi)

To cite this article:

Suchuan Zhang, Qiao Shi. Linking Protestant Work Ethic to Workplace Ostracism: The Mediating Effect of Subjective Well-Being. Journal of Investment and Management. Vol. 6, No. 2, 2017, pp. 66-74. doi: 10.11648/j.jim.20170602.12

Received: May 26, 2017; Accepted: June 19, 2017; Published: July 20, 2017

\begin{abstract}
In this study, the present paper examined the relationship between Protestant Work Ethic (PWE) and Workplace Ostracism by focusing on the mediating effect of Subjective Well-Being (SWB) which is a construct with three components that include life satisfaction, positive affect and negative affect. Our theoretical model was tested using data collected from employees in different firms from different provinces in China. Analyses of multisource and lagged data from 677 employees indicated that as predicted, life satisfaction, positive affect and negative affect mediate the relationship between PWE and Workplace Ostracism. Theoretical and practical implications are discussed.
\end{abstract}

Keywords: Protestant Work Ethic, Subjective Well-Being, Workplace Ostracism

\section{Introduction}

Weber (1905) first proposed the Protestant Work Ethic (PWE) as a cultural value that represents the extent to which individuals place work at or near the center of their lives [1]. The beliefs in PWE straddle nearly all of the social sciences especially psychology and sociology [2] while it appears, and it has embedded in popular culture and been acknowledged as a crucial determinant of work-related behaviors [3]. It is still invite scholarly interests in such diverse fields today. However, relatively little recent attention has been given to the concept of PWE in the applied psychological and management literature assuming that it's necessary to explore the relations of PWE to organizationally relevant variables in the workplace.

Scholarly work has identified that there is a pervasive and universal phenomenon at workplace called Workplace Ostracism [4]. Workplace Ostracism refers to individuals being excluded and ignored by others in workplace [5]. And it associated with diminished psychological well-being and satisfaction with colleagues [6] [7]. Being excluded and ignored in workplace is an unpleasant, even painful experience. What's more, not only are such experiences painful, but under some circumstances they can have an even greater negative impact than other harmful workplace behaviors such as aggression and harassment. There is a large body of literature about Workplace Ostracism. Most researchers focus on the detrimental consequences of ostracism in the workplace [8], such as presenting challenges that can lessen the resources individuals hold [9] which cause employees experience emotional exhaustion [10] and deteriorated psychological well-beings [11]; associating with undesirable behaviors, examples as acting rudely, withholding assistance and mocking with others [12]; increasing individuals' job stress and turnover intention and decreasing job performance [9]. There is little concern about the factors that precipitate the occurrence of this phenomenon [13]. Just as $\mathrm{Wu}$, Wei, and Hui (2011) [11] note that examinations of the antecedents of Workplace Ostracism is indeed significant, on account of only when the antecedents are designated, can efficacious measures be taken to minimize the occurrence of Workplace Ostracism.

Given ostracism's frequency and impact, understanding how to cope with ostracism is also critical because effective coping strategies may mitigate workplace ostracism. Recent research on positive organizational behavior focused on employee well-being in the workplace [14]. Employee well being is an important variable in coping with Workplace Ostracism. Bandura (1986) [15], for example, claimed that employee Well-Being is a function of aversive experiences at workplace (for example: depression, anxiety, emotional exhaustion and job tension). However, Diener (1999) [16] 
states that Subjective Well-Being (SWB) is essential to well-being. Moreover, findings to date suggest that employees with high SWB are happier and more satisfied with their lives, and they are more likely to be effective workers and to have successful careers [17]. SWB is defined as a person's evaluation of his or her life, The study of SWB is a new area in the behavioral sciences, and it has been empirically shown that a happy worker is more likely to be successful in many aspects of life. More importantly, the importance of SWB is crucial since empirically it has been shown that a happy worker is more likely to be successful in many aspects especially in workplace. Thus, the main objective of present article is to examine the relationship of PWE with Workplace Ostracism by focusing the mediating effect of SWB.

\section{Literature and Hypotheses}

\subsection{Subjective Well-Being (SWB)}

Diener, Oishi and Lucas (2015) [18] defined SWB as individuals' evaluations of their lives - the degree to which their thoughtful appraisals and affective reactions indicate that their lives are desirable and proceeding well. It is a multidimensional construct that includes cognitive and affective Components. Specifically, Diener (1984, 2000) [19] [20] defined SWB as a construct with three components which include life satisfaction, positive affect or the frequent experiences of pleasant emotions such as feelings of energy and engagement, and a low level of negative affect or a relative absence of unpleasant emotions such as distress and anxiety. Subsequent empirical studies supported this tripartite structure of SWB [21] [22]. Furthermore, these three components are related but separable constructs [19], and can be easily measured using self-reports in a valid and reliable manner [23] [24].

\subsection{PWE, Life Satisfaction and Workplace Ostracism}

The Protestant Work Ethic (PWE), an orientation to work that is a set of beliefs and values that work is valuable, important, and a central life interest [25]. Blood (1969) [26] stated that the more an individual endorse PWE beliefs the more satisfied they were with their lives in general. In addition, Furnham (1984) [27] demonstrated that congruent environments for the subjects who endorse PWE are among individuals with similar tastes and values to their own, and they can perform tasks which they enjoy and are able to do, these environments provides life satisfaction for them. These studies have analyzed the relationship between PWE and life satisfaction. Life satisfaction is the cognitive component of SWB [28], and this component is a cognitive judgmental evaluation of one's life [29]. The relationship between PWE and life satisfaction have been demonstrated by many other studies except above review. For example, Emmons (1986) [30] found that the strivings to work predicted life satisfaction, relevantly, the person with high PWE is respect for work, and willingness to make an effort to work [31]. Except for that,
Ghorpade, Lackritz and Singh (2006) [32] stated that self-esteem is personality traits that is initially correlates to PWE, and self-esteem is one of the strongest predictors of life satisfaction [33].

Workplace Ostracism is the degree to which individuals perceive that they are ignored or excluded by other employees at workplace [8]. Several descriptions of Workplace Ostracism behaviors have been offered, for example, withholding needed information, giving the silent treatment, avoiding conversation or eye contact, and giving the cold shoulder [5], as well as undesirable behaviors such as acting rudely, mocking or arguing with others, and withholding assistance [12]. Moreover, Workplace Ostracism is demonstrated is associate with anxiety, depression and stress [18] [7]. However, there are many study state that life satisfaction is negatively correlated with depression, anxiety, emotional distress, anger, loneliness, and symptoms of psychological disorders [34]. In addition to these, individuals with lower life satisfaction are at risk for a variety of psychological and social problems such as maladaptive relationships with others [35]. Obviously, these theories have shown a negative correlation between life satisfaction and Workplace Ostracism. In the terms of these psychological impact of lower life satisfaction, a variety of studies have examined these various outcomes associate with and go with the Workplace Ostracism [36]. More specifically, Wu et al. (2011) [11] proved that the person with lower life satisfaction is particularly at higher risk of being ostracized. These above reasons allow for a clearer conceptual understanding of how life satisfaction mediates the relationship of PWE to Workplace Ostracism, we propose:

Hypothesis 1: life satisfaction mediates the relationship between PWE and Workplace Ostracism.

\subsection{PWE, Positive Affect, Negative Affect and Workplace Ostracism}

Locus of control, self-esteem and self-efficacy are the personality traits of individuals with PWE beliefs [37] [38] [39]. And individuals with high PWE beliefs experience a sense of personal engagement in work-related tasks [40]. However, a large body of studies shows that these personality traits are the source of positive affect (PA) and can lessen the feeling of negative affect (NA) [19]. PA and NA are the affective component of SWB. Watson, Clark \& Tellegen (1988) [41] stated that PA reflects the extent to which a person feels enthusiastic, active, and alert; NA is unpleasurable engagement that subsumes a variety of aversive mood states. Just as above literature review, researches show that PWE is strongly related to PA and NA. For example, Brandt (1980) [42] found that locus of control is related to PA in a number of populations. Reich and Zautra (1981) [43] postulate that self-efficacy is a ubiquitous source of PA. And Diener (1984) mentioned that commitment and involvement can lessen negative emotions [19].

There are a large number of studies reporting on the relationship of PA, NA and Workplace Ostracism. Costa and McCrae (1995) [44] suggest that extraversion is one basic 
dimension of personality that lead to PA. In addition, extraversion was found to co-vary with PA [45] [46]. Consequently, Wu et al. (2011) examined how extroversion relates to Workplace Ostracism [11], and finally found support for the idea of extroversion is negatively related to Workplace Ostracism. Except for these, there is some other empirical support for the notion that NA is positively relate to Workplace Ostracism. NA subsumes a variety of aversive mood states such as anger, contempt, disgust, guilt, fear, and nervousness [41]. And these mood states accompany the Workplace Ostracism behaviors such as acting rudely, mocking or arguing with others, and withholding assistance [12]. In addition to these, George (1992) [47] reported employees who exhibit high NA have worse relationship with co-workers and supervisors, and it has been found to be associated with ostracism at workplace [48] [49] . On the basis of our literature review, we propose:

Hypothesis 2: PA mediates the relationship between PWE and Workplace Ostracism.

Hypothesis 3: NA mediates the relationship between PWE and Workplace Ostracism.

\section{Method}

\subsection{Sample and Procedures}

Participants in this study were employees from different enterprises in different provinces in China, such as Beijing, Inner Mongolia Autonomous Region, Guangdong, Fujian, Shanxi, etc.

Survey data were collected at two time points separated by 3 Months. And two waves of data collection were performed in order to reduce the common method bias [50]. In the first-wave survey $\left(T_{1}\right)$, the employees' information on their own demographics, PWE and SWB were provided. In the second-wave survey $\left(T_{2}\right.$, three months after $\left.T_{1}\right)$, the employees reported their perceptions of Workplace Ostracism.

Data were collected based on the following procedures. With the assistance of the human resource managers of firms, a list of 1300 randomly selected employees was prepared. Survey questionnaires were coded before distribution, and the human resources departments assisted in matching the identity numbers and names of respondents with the responses of the $\mathrm{T}_{1}$ and $\mathrm{T}_{2}$ surveys conducted. Questionnaires were then administered to the selected employees separately. The employees acquired the survey packets in a meeting room during work hours, in the presence of data collectors trained and led by one of the authors. Respondents were informed that the survey aimed to examine the experience of human resource practices; they were assured of the confidentiality of their responses. Each respondent placed his or her completed survey into a sealed envelope, and returned it to a box positioned in the human resources department. Survey questionnaires were coded before distribution. The scales were converted into Chinese following the commonly used back translation procedure [51].

In wave one, 810 questionnaires were returned, with a response rate of $62.3 \%$. Three months later, the second-wave survey was conducted, and 684 questionnaires were returned, with a response rate of $84.4 \%$. In total, after questionnaires with missing data were eliminated, there were 667 usable responses. A total of 363 were females (54.4\%); 329 were in the age range of $25-34(49.3 \%) ; 330$ were in the tenure range of $3-5(49.5 \%)$. The employees have a good educational background with a percentage of $82.2 \%$ above college level. The jobs of these employees involved relatively high levels of social interactions. Thus, it appears that this sample is relevant and suitable for testing our theoretical model.

\subsection{Instruments}

\subsubsection{Protestant Work Ethic}

Mirels and Garrett's (1971) 19-item scale was used to measure protestant work ethic, with each item rated on a 5-point Likert-type scale. The scale is the most frequently used in the literature [52] and continues to be employed in research studies [53] [54] [55] to assess the PWE construct to date. Sample items are, "Life would be more meaningful if we had more leisure time." (Reverse coded), "There are few satisfactions equal to the realization that one has done his best at a job." "The most people who do not succeed in life are just plain lazy." "Any man who is able and willing to work hard has a good chance of succeeding." "Hard work offers little guarantee of success." Cronbach's alpha for the scale was 0.86 $($ mean $=62.81, \mathrm{SD}=10.15)$.

\subsubsection{Subjective Well-Being}

The SWB scales are designed to measure global evaluations of affect and life quality. SWB has three major components, including one cognitive dimension (i.e., life satisfaction) two affective dimensions (i.e., positive affect and negative affect). Life satisfaction was assessed with the Satisfaction with Life Scale [56]. The most widely used SWLS is a five-item self-report measure of overall satisfaction with life, with responses measured on a Likert scale ranging from 1 (strongly disagree) to 5 (strongly agree). This scale measures life satisfaction by asking a person to provide an overall judgment of their quality of life. Sample items are, "In most ways my life is close to my ideal." "The conditions of my life are excellent." "I am satisfied with my life." "So far I have gotten the important things I want in life." "If I could live my life over, I would change almost nothing." Cronbach's alpha for this measure was $0.80($ mean $=16.52, \mathrm{SD}=3.40)$.

Affect dimension was assessed using the Positive and Negative Affect Schedule [41]. The Positive affect scale consists of active, alert, attentive, determined, enthusiastic, excited, inspired, interested, proud, and strong (Cronbach's alpha $=0.89)$. The Negative affect scale is comprised of afraid, ashamed, distressed, guilty, hostile, irritable, jittery, nervous, scared, and upset (Cronbach's alpha $=0.87$ ). Participants rated the extent to which they had felt each of the affects "during the past few days".

\subsubsection{Workplace Ostracism}

Employees completed the 10-item Workplace Ostracism 
scale developed by Ferris et al. (2008) which assesses exclusionary behavior. Sample items included: "Others avoided you at work," "Others at work shut you out of the conversation." "Others refused to talk to you at work." "Others left the area when you entered" "Your greetings have gone unanswered at work." "You involuntarily sat alone in a crowded lunchroom at work." and "Others at work treated you as if you weren't there." The scale's reliability was 0.93 (mean $=20.56, \mathrm{SD}=7.63)$.

\subsubsection{Control Variable}

In line with previous work, this study controlled for the employee's gender, as females more often report being the target of workplace incivility [57] and aggressive behavior [58]. Both the social psychological and organizational literature suggest that age is related to the occurrence of workplace exclusion, thus, age is controlled for in this study. The paper also controlled for the tenure and education because of the possibility that employees newer who have had less time to develop meaningful work relationships may report levels of exclusion than those with more years of tenure [13] and one's level of education is associated with substance abuse [59] which often associated with acts of exclusion while at work. Finally, we controlled for the income because of Diener, Louis, and Shigehiro (2013) [60] found that rising household income is significantly associated with all three forms of subjective well-being.

\section{Results}

\subsection{Confirmatory Factor Analysis}

Confirmatory factor analysis (CFA) was conducted to ensure sufficient convergent and discriminant validity among all variables in our model. We first tested a model that consisted of five factors: PWE, life satisfaction, PA, NA and Workplace Ostracism. Results showed the five-factor model fit the data well $\left(\chi^{2}=951.26, d f=220, p<0.001\right.$, RMSEA $=0.07, \mathrm{CFI}=$ $0.91, \mathrm{TLI}=0.90)$. We further compared the five-factor model to an alternative three-factor model, which included PWE as a single factor, the mediator variables (life satisfaction, PA and NA) as a single factor, Workplace Ostracism as a separate factor $\left(\chi^{2}=623.83, d f=62, p<0.001, \mathrm{RMSEA}=0.12, \mathrm{CFI}=0.85\right.$, TLI $=0.81)$. Finally, we compared five-factor model to a one-factor model, in which all items loaded onto a single factor $\left(\chi^{2}=277.01, d f=5, p<0.001, \mathrm{RMSEA}=0.29, \mathrm{CFI}=0.05, \mathrm{TLI}\right.$ $=0.28$ ). Model comparison results revealed that the five-factor model fitted the data considerably better than any of the alternative models. Given these results, all five constructs were applied in subsequent analyses.

\subsection{Descriptive Statistics}

Table 1 presents the means, standard deviations, and zero-order Pearson correlations of all key variables.

Table 1. Means, standard deviations, correlations and reliabilities of study variables.

\begin{tabular}{|c|c|c|c|c|c|c|c|c|c|c|}
\hline Variables & 1 & 2 & 3 & 4 & 5 & 6 & 7 & 8 & 9 & 10 \\
\hline \multicolumn{11}{|l|}{ 1. Gender } \\
\hline 2. Age & $0.08 *$ & & & & & & & & & \\
\hline 3. Education & $-0.11 * *$ & $-0.11 * *$ & & & & & & & & \\
\hline 4. Income & 0.07 & $0.15 * *$ & -0.07 & & & & & & & \\
\hline 5. Job tenure & 0.02 & $0.28 * *$ & $-0.28 * *$ & $0.21 * *$ & & & & & & \\
\hline 6. PWE & $-0.08 *$ & 0.07 & 0.056 & 0.07 & $0.08^{*}$ & $(0.86)$ & & & & \\
\hline 7. Life satisfaction & $-0.10 * *$ & $0.15 * *$ & -0.03 & -0.01 & $0.17 * *$ & $0.33 * *$ & $(0.80)$ & & & \\
\hline 8. Positive affect & -0.04 & $0.10 *$ & 0.04 & -0.00 & 0.00 & $0.45^{* *}$ & $0.42 * *$ & $(0.89)$ & & \\
\hline 9. Negative affect & 0.04 & -0.06 & 0.02 & -0.05 & $-0.19 * *$ & $0.22 * *$ & $-0.13 * *$ & $0.09 *$ & $(0.87)$ & \\
\hline 10. Workplace ostracism & $0.08 *$ & -0.05 & 0.03 & $-0.08^{*}$ & $-0.10 * *$ & $0.09 *$ & $-0.02 *$ & $-0.03 *$ & $0.51 * *$ & $(0.93)$ \\
\hline$S D$ & 0.50 & 1.43 & 0.56 & 0.76 & 1.03 & 0.53 & 0.68 & 0.76 & 0.73 & 0.76 \\
\hline
\end{tabular}

Notes. Values in parentheses on the diagonal are the Cronbach's alpha value of each scale. $\mathrm{n}=667 ; * \mathrm{p}<0.05$, two tailed, $* * \mathrm{p}<0.01$, two tailed.

As shown, PWE is positively correlated with life satisfaction $(r=0.33, p<0.01)$, positive affect $(r=0.45, p<$ $0.01)$, and negative affect $(r=0.22, p<0.01)$. In addition, life satisfaction $(r=-0.02, p<0.05)$ and positive affect $(r=-0.03$, $p<0.05)$ are negatively correlated with Workplace Ostracism, negative affect $(r=0.51, p<0.01)$ is positively correlated with Workplace Ostracism. These results support the hypotheses.

\subsection{Hypothesis Testing}

The hypotheses predict that life satisfaction, positive affect and negative affect, the three component of SWB mediate the relationship between PWE and Workplace Ostracism. In order to test our multiple-mediation model, we followed a structural equation modeling (SEM) approach using Amos 17.0. Figure 1 displays the structural equation model. 


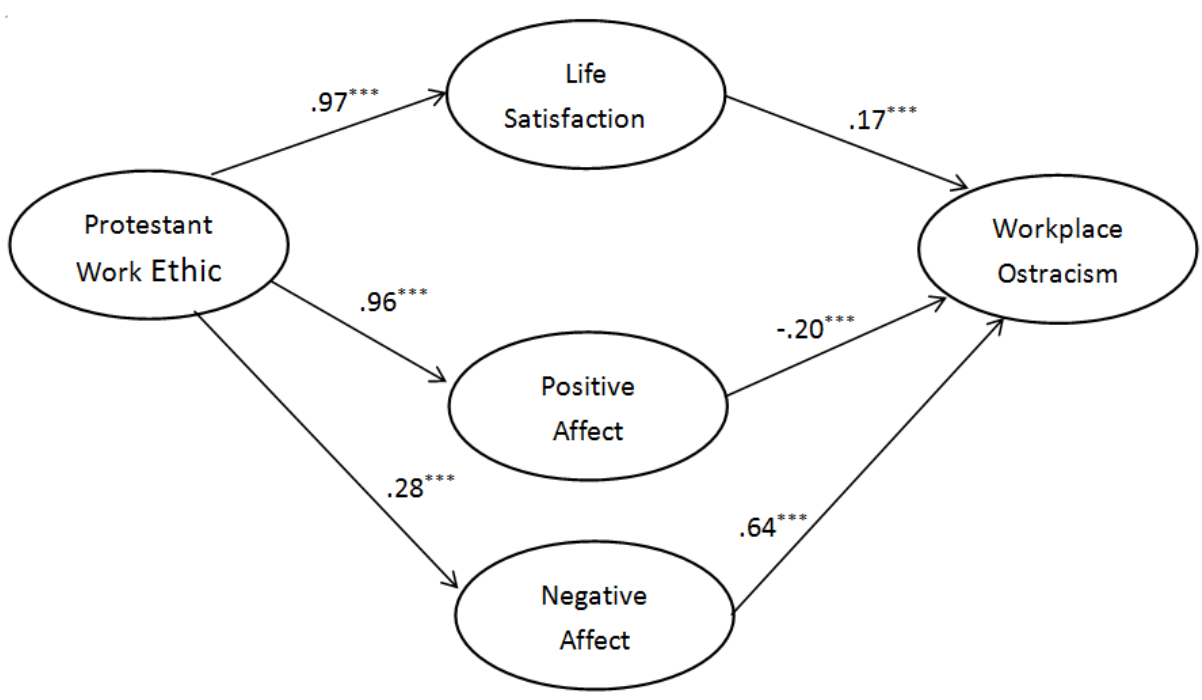

Figure 1. Structural Equation Model (Full Mediation).

Results show that the model had a good fit to the data $\left(\chi^{2}=\right.$ $1198.57, d f=224, p<0.001$, RMSEA $=0.07, \mathrm{CFI}=0.91$, TLI $=0.91)$. To further examine whether the three component of SWB fully or partially mediate the relationship between PWE and Workplace Ostracism, we tested an alternative model that included direct paths from the independent variable to the outcome variables. This partial mediation model also provided a good fit to the data $\left(\chi^{2}=1198.25, d f=223, p<0.001\right.$,
RMSEA $=0.08$, CFI $=0.90$, TLI $=0.90$ ). Results show non significant direct path from PWE to Workplace Ostracism $(\beta=$ $-0.17, p \geq 0.05$ ). Besides, as this results show, full mediation model offered a slightly better fit than the partial mediation model, indicating that the three component of SWB fully mediate the relationship between PWE and Workplace Ostracism (see Figure 2). Thus, H1, H1 and H1 are supported.

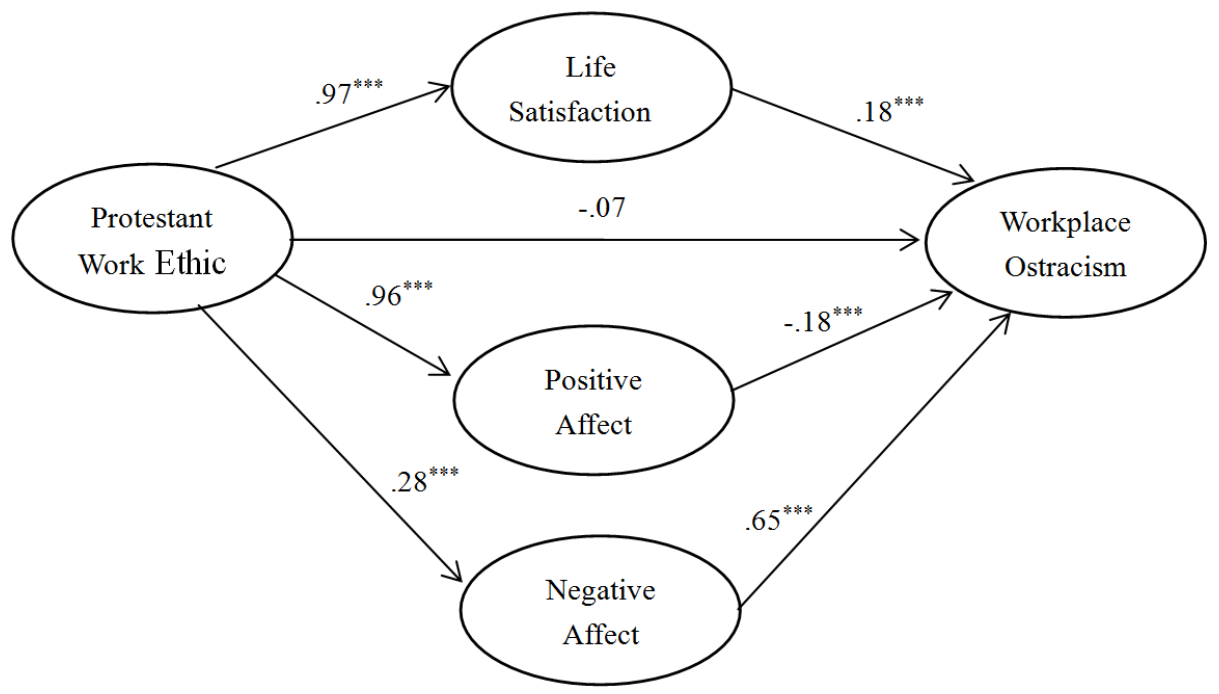

Figure 2. Structural Equation Model (Partial Mediation).

\section{Discussion}

This study provides a test of the mediating mechanisms by which PWE impacts Workplace Ostracism. The results demonstrate that each of the hypothesized mediation mechanisms plays a significant role in explaining the impact of PWE on Workplace Ostracism. Life satisfaction, the cognitive component of SWB, was found mediate the relationship between PWE and Workplace Ostracism. Second, positive affect and negative, the affect component of SWB, were found mediate the relationship between PWE and Workplace Ostracism.

This work demonstrates that individuals' perceptions of SWB mediate the relationship between PWE and Workplace Ostracism. First, we support the idea that life satisfaction is a mediator. This finding is consistent with the previous researches, which has concluded that PWE relates to life satisfaction, and life satisfaction affects Workplace Ostracism. A look back on the history of the PWE construct might be useful in explaining this result. The result of relationship between PWE and life satisfaction is in line with findings of 
Buzkurt et al. (2010) [61] who reported that high endorsement PWE employees derive life satisfaction by putting enough effort and labor to earn it when given a task. To some extent, this is consistent with the popular saying that "To live like a king, one has to work like slave." In the case of PWE, it is an orientation to work and a set of beliefs that work is valuable, important, and a central life interest [25], as a result of it, employees with high PWE are more motivated for hard work [62], so forth in order to build their organization with high quality of life, which means perceive high life satisfaction in their organization [63]. Similarly, this result also goes in line with a previous research, which had concluded that employees who scored high on PWE tended to work hard to produce and build quality in their work and will enjoy the satisfaction that comes out of their sweat as they get identified with good workmanship [64]. As the report also demonstrated that employees with high life satisfaction are at less risk of being ostracism, this is consistent with Boehm and Lyubomirsky (2008) [65] theorizing that person perceive high life satisfaction tend to be more popular and likable. When participant responses to individual items, such as "others avoided you at work" or "others refused to talk to you at work", the result is examined. On the other hand, it also in line with Diener's (2012) theory that high life satisfaction person seems to foster success in the workplace. As we all know, individuals are generally expected to work together to achieve common organizational goals at organizational setting [66]. So that when their co-workers perceive them to be highly valuable or especially relevant to their future success, employees are less likely to become the target of ostracism.

Second, the present paper identify that PA mediate the effect of PWE on Workplace Ostracism. This result confirms that person with high PWE will feel high PA. And the result is in accordance with study of Higgins et al., (2001), PWE endorsement may be associated with what called a prevention focus rather than a promotion focus, that is, high PWEs may be more concerned with striving for pleasant end states (e.g. enthusiastic, active). More specifically, we can learn from Furnham (1990b) that employees with PWE are better workers and are more likely to achieve more while at work and will feel high positive emotions while at work [31] [67]. The finding of the negative relationship between PA and Workplace Ostracism is consistent with existing literature [68]. Diener (2012) claimed that positive feelings help people in a number of areas of their lives, such as leading to better relationship and interaction at workplace. In addition, he also demonstrated that employee with high PA are likely to be more productive, creative and efficient, as we all know, these characters are likable and popular at workplace [64]. Just as the example of Google's offices, in which the company lets employees feel truly comfortable, even with positive feeling. Shin et al. (2011) stated that PA is associated with peer acceptance and initiating positive interaction with peers. So if you feel high PA, you get more chance to communicate and cooperate with co-workers and get less chance of perceiving ostracism. In sum, the findings demonstrated that PA mediates the relationship between PWE and Workplace Ostracism.
Furthermore, this paper demonstrates the mediate role of NA. PA and NA are two reverse emotions. Person feels high levels of PA, which means lower in NA. Person with high PWE achieve more in hard working, feel high in PA and low in NA because of work is the central interest of their life [25]. When at workplace, person with PWE beliefs felt low in NA because they can attain goals of their life. This confirms the present finding which presented the negative relationship between PWE and NA. Taken together, these consistence with Higgins et al. (2001) stating that employees with high PWE may be more concerned with avoiding unpleasant end states (e.g. Depression, anxiety). Worth noting, the result of the effect of NA and Workplace Ostracism presented by this paper in a way agree with previous research where the employees who display negative behaviors at work are more likely to become the target of mistreatment [69]. Specifically, NA contains a variety of unpleasurable mood states, such as anxiety, anger, depress and so on. When person around you presence these mood, you will not like he or she, and avoid them even not cooperate with him or her. Then Workplace Ostracism will happen. Therefore, employees with high NA are more likely to be targeted of Workplace Ostracism. This finding is consistent with Diener (2012) theorizing that when people meet others who are depressed, they tend to want to avoid them in workplace. In considering this result, it is important to note that managers nowadays minimize the occurrence of Workplace Ostracism by decreasing employees' NA such as depression and stress. Moreover, neuroticism is always associated with NA, so the person feel NA at work are at risk of ostracism because Leung et al (2011) [70] demonstrated that neurotic individuals are more likely to perceive ostracism. On the other hand, employees who are viewed as neurotic may be at risk for exclusion, as neurotic could be construed as unstable or unreliable from a relational standpoint [58]. All the present results of this paper confirmed the above view: NA mediate the relationship between PWE and Workplace Ostracism.

\subsection{Contributions and Implications}

The present research makes several distinct theoretical contributions. First, the paper built and empirically examined a conceptual model that links PWE to Workplace Ostracism by focusing the mediating effects of SWB. The final results empirically demonstrated that PWE is one determinant of SWB. Specifically, we proved that who have a strong commitment to working hard, admiration of work itself, asceticism, negative attitudes to leisure and internal motive, present higher level of life satisfaction, PA and lower NA. Such results devote to the SWB research by indicating some critical antecedents.

Second, going beyond previous correlation analysis of the emotional antecedents of Workplace Ostracism [11], the present study applied the regression analysis to the relationship between SWB and Workplace Ostracism, and found that life satisfaction and positive affect are negatively related to Workplace Ostracism, negative affect is positively related to Workplace Ostracism. This indicates that subjects 
who present high life satisfaction, positive affect and low negative affect are less likely being the target of Workplace Ostracism.

Third, extending this theoretical implication of our findings, it is also possible that SWB may be used to cope not only with Workplace Ostracism, but also with other destructive behaviors such as abusive supervision [11].

Fourth, the pattern of full mediation results also points to future research opportunities. The impact of PWE on Workplace Ostracism was fully mediated by life satisfaction, PA and NA, the three component of SWB. Thus, these direct influence of PWE and Workplace Ostracism may be worthy of further examination. Moreover, these results suggest the effects of PWE on Workplace Ostracism may operate through additional mediation paths. In general, the consideration of SWB opens new avenues for Workplace Ostracism and PWE research.

This theoretical model and empirically findings also have crucial practical implications. As Wu et al. (2011) stated that that Workplace Ostracism is costly for both employees and organizations. Our findings suggest that managers or leaders can strengthen employees perception of SWB by offering programs and office events that encourage interaction and communication [71] to lessen the occurrence of Workplace Ostracism. Moreover, as our findings show that employees low in life satisfaction and positive affect are more likely to gain perception of ostracized. Hence, managers or leaders should pay attention to these employees in particular, simultaneously provide training, counseling, and social support for them so as to help them keep away from Workplace Ostracism and decrease the negative impact of it.

\subsection{Strengths and Limitations}

The present study has multiple strengths worth mentioning. First, two waves of data collection from different sources decrease the potential problems associated with the common method variance as Podsakoff et al. (2003) mentioned. Second, collected data at three distinct points in time lends some support to the causal nature of the model with regard to the antecedents of Workplace Ostracism [72].

The study has a limitation that should be noted. A constraint on the generalizability of our findings is Chinese collectivistic cultures. As Hui (1988) [73] demonstrated that Collectivism is a series of feelings, beliefs, behavioral intentions, and manners related to solidarity and concern for others. Collectivistic cultures stress on close and harmonious interpersonal relationships. As a result, employees will be more likely to be sensitive to Workplace Ostracism in collectivistic cultures [74]. The present findings may not be summarized in more individualistic cultures on account of our data were collected in China.

\section{Conclusion}

Drawing on the mediating effect of Subjective Well-Being (SWB) which is a construct with three components that include life satisfaction, positive affect and negative affect, this research examined the relationship between Protestant Work Ethic (PWE) and Workplace Ostracism. Result indicated that as predicted, life satisfaction, positive affect and negative affect mediate the relationship between PWE and Workplace Ostracism.

Ethical approval: All applicable international, national, and/or institutional guidelines for the care and use of animals were followed.

\section{References}

[1] Furnham, A. (1990a). A content, correlational, and factor analytic study of seven questionnaire measures of the Protestant work ethic. Human Relations, 43, 383-399.

[2] Furnham, A. F. (1984). The protestant work ethic: A review of the psychological literature. European Journal of Social Psychology, 14, 87-104.

[3] Michael J. Miller, David J. Woehr \& Natasha Hudspeth (2001) The Meaning and Measurement of Work Ethic: Construction and Initial Validation of a Multidimensional Inventory. Journal of Vocational Behavior 59, 1-39.

[4] Ferris, D. L., Brown, D. J., Berry, J. W. and Lian, H. (2008). The development and validation of the Workplace Ostracism scale. Journal of Applied Psychology, 93, 1348-1366.

[5] Williams, K. D., (2001). Ostracism: The Power of Silence. Guilford Press, New York.

[6] Baumeister, R. F., \& Tice, D. M. (1990). Anxiety and social Exclusion. Journal of Social and Clinical Psychology, 9, 165195.

[7] Hitlan, R. T., Cliffton, R. J., \& DeSoto, M. C. (2006). Perceived exclusion in the workplace: The moderating effects of gender on work-related attitudes and psychological health. North American Journal of Psychology, 8 (2), 217-236.

[8] Ferris, D. L., Brown, D. J., Berry, J. W. and Lian, H. (2008). The development and validation of the Workplace Ostracism scale. Journal of Applied Psychology, 93, 1348-1366.

[9] Haq, I. U. (2014). Workplace Ostracism and job outcomes: Moderating effects of psychological capital. International Conference, 7, 25-27.

[10] Lee, R. T. and Ashforth, B. E. (1996). A meta-analytic examination of the correlates of the three dimensions of job burnout. Journal of Applied Psychology, 81, 123-133.

[11] Wu, L. Z., Wei, L. Q., Hui, C., (2011). Dispositional antecedents and consequences of Workplace Ostracism:an empirical examination. Frontiers of Business Research in China 5 (1), 23-44.

[12] Thau, S., Aquino, K., \& Poortvliet, P. M. (2007). Self-defeating behaviors in organizations: The relationship between thwarted belonging and interpersonal work behaviors. Journal of Applied Psychology, 92, 840-847.

[13] Kristin L. Scott, Simon Lloyd D. Restubog, Thomas J. Zagenczyk (2013). A social exchange-based model of the antecedents of workplace exclusion. Journal of Applied Psychology, 98 (1), 37-48. 
[14] Luthans, F., Youssef, C. M., Avolio, B. J. (2007). Psychological capital. New York: Oxford University Press.

[15] Bandura, A. (1986). Social foundations of thought and action: A social cognitive theory. Englewood Cliffs, NJ: Prentice-Hall.

[16] Diener, E., Sun, E. M., Lucas, R. E., \& Smith, H. L. (1999). Subjective Well-Being: Three decades of progress. Psychological Bulletin, 125, 276-302.

[17] Tay, Kuykendall \& Diener. (2015). Satisfaction and happiness-The bright side of quality of life. In Global Handbook of Quality of Life (pp. 839-853). Springer Netherlands.

[18] Diener, E., Shigehiro Oishi \& Richard E. Lucas. (2015). National accounts of Subjective Well-Being. American Psychologist, 70 (3), 234-242.

[19] Diener, E. (1984). Subjective Well-Being. Psychological Bulletin, 95 (3), 542-575.

[20] Diener, E. (2000). Subjective well-being: The science of happiness and a proposal for a national index. American Psychologist, 55, 34-43.

[21] Arthaud-Day, M. L., Rode, J. C., Mooney, C. H., \& Near J. P. (2005). The subjective well-being construct: A test of its convergent, discriminant, and factorial validity. Social Indicators Research, 74, 445-476.

[22] Diener, E., Oishi, S., \& Lucas, R. E. (2003). Personality, culture, and subjective well-being: Emotional and cognitive evaluations of life. Annual Review of Psychology, 54, 403-425.

[23] Diener, E., Inglehart, R., \& Tay, L. (2013). Theory and validity of life satisfaction scales. Social Indicators Research, $112,497-527$.

[24] Schimmack, U., \& Oishi, S. (2005). The influence of chronically and temporarily accessible information on life satisfaction judgments. Journal of Personality and Social Psychology, 89, 395-406.

[25] Sung Ho, K. (2008). Max Weber. The Stanford Encyclopedia of Philosophy. Metaphysics Research Lab, CSLI, Stanford University. Retrieved 21 August. 2011.

[26] Blood, M. (1969). Work values and job satisfaction, Journal of Applied Psychology, 53, 456-469.

[27] Furnham, A. F. (1984). The protestant work ethic: A review of the psychological literature. European Journal of Social Psychology, 14, 87-104.

[28] Diener, E., Derrick Wirtz, William Tov, Chu Kim-Prieto, Dongwon Choi, Shigehiro Oishi \& Robert Biswas Diener (2010). New Well-being measures: Short scales to assess flourishing and positive and negative feeling. Soc Indic Res, 97, 143-156.

[29] Diener, E., Sandvik, E., \& Pavot, W. (2009). Happiness is the frequency, not the intensity, of positive versus negative affect. Social Indicators Research Series, 39, 213-231.

[30] Emmons, R. A. (1986). Personal strivings: An approach to personality and Subjective well-being. Journal of Personality and Social Psychology. 51, 1058-1068.

[31] Furnham, A. (1990b). The Protestant work ethic and type a behavior. Psychological Records, 66, 323-328.

[32] Ghorpade, Lackritz \& Singh. (2006). Correlates of the
Protestant Ethic of Hard Work: Results From a Diverse Ethno-Religious Sample. Journal of Applied Social Psychology, 36, 10, pp. 2449-2473.

[33] Drumgoole, W. P. (1981). Self-concept and life satisfaction as perceived by young, middle-aged, and senior adults (Doctoral dissertation, East Texas State University, 1980). Dissertation Abstracts International, 41, 2939A. (University Microfilms No. 80-27, 666).

[34] Huebner, E. S. (1991). Correlates of life satisfaction in children. School Psychology Quarterly, 6, 103-111.

[35] Furr, R. M., and D. C. Funder. (1998). A multimodal analysis of personal negativity. Journal of Personality and Social Psychology, 74, 1580-1591.

[36] Baumeister, R. F., Twenge, J. M., \& Nuss, C. K. (2002). Effects of social exclusion on cognitive processes: anticipated aloneness reduces intelligent thought. J. Personal. Soc. Psychol. 83, 817-827.

[37] Furnham, A. F. (1987). Predicting protestant work ethic beliefs. European Journal of Personality, Vol. 1, 93-106.

[38] Mirels, H., \& Garrett, J. (1971). Protestant ethic as a personality variable. Journal of Consulting and Clinical Psychology, 36 (1), 40-44.

[39] Sarah S. M. Townsend \& Leigh L. Thompson. (2014). Implications of the Protestant work ethic for cooperative and mixed-motive teams. Organizational Psychology Review Vol. 4 (1), 4-26.

[40] Tang, T. L. P., Singer, M. G., \& Roberts, S. (2000). Employees' perceived organizational instrumentality: An examination of the gender differences. Journal of Managerial Psychology, 15, $378-406$.

[41] Watson, D., Clark, L. A., \& Tellegen, A. (1988). Development and validation of brief measures of positive and negative affect: The PANAS scales. Journal of Personality and Social Psychology, 54, 1063-1070.

[42] Baron, R. M., \& Kenny, D. A. (1986). The moderator-mediator variable distinction in social psychological research: Concepual, strategic, and statistical considerations. Journal of Personality and Social Psychology, $51,1173-1182$.

[43] Reich, J. W., \& Zautra, A. (1981). Life events and personal causation: Some relationships with satisfaction and distress. Journal of Personality and Social Psychology, 41, 1002-1012.

[44] Costa, P. T., \& McCrae, R. R. (1995). Primary traits of Eysenck P-E-N system-3-factor and 5-factor solutions. Journal of Personality and Social Psychology, 69, 308-317.

[45] Gorman, B. S. (1972). A multivariate study of the relationships of cognitive control and cognitive style principles to reported daily mood experiences. (Doctoral dissertation, City University of New York, 1971 ). Dissertation Abstracts International, 32, 4211B. (University Microfilms, No. 72-5071).

[46] Tolor, A. (1978). Personality correlates of the joy of life. Journal of Clinical Psychology. 34, 671-676.

[47] George, J. M. (1992). The role of personality in organizational life: Issues and evidence. Journal of Management, 18, 185-213. 
[48] Eysenck H., \& Gudjonsson G. (1989). The causes and cures of criminality. New York: Plenum.

[49] Heaven P. (1996). Personality and self-reported delinquency: Analysis of the Big Five personality dimensions. Personality and Individual Differences, 20, 47-54.

[50] Podsakoff, P. M., MacKenzie, S. B., Lee, J. S., \& Podsakoff, N. P. (2003). Common method biases in behavioral research: A critical review of the literature and recommended remedies. Journal of Applied Psychology, 88, 879-903.

[51] Brislin, R. W. (1980). 'Translation and content analysis of oral and written materials'. In Triandis, H. C. \& Berry, J. W. (Eds), Handbook of Cross-Cultural Psychology (Vol. 2, 389-444). Boston, MA: Allyn \& Bacon.

[52] Miller, M. J., Woehr, D. J., \& Hudspeth, N. (2002). The meaning and measurement of work ethic: Construction and initial validation of a multidimensional inventory. Journal of Vocational Behavior, 60 (3), 451-489.

[53] Li, Y. J., Johnson, K. A., Cohen, A. B., Williams, M. J., Knowles, E. D., \& Chen, Z. (2012). Fundamental (ist) attribution error: Protestants are dispositionally focused. Journal of Personality and Social Psychology, 102, 281-290.

[54] Linderbaum, B. A., \& Levy, P. E. (2010). The development and validation of the Feedback Orientation Scale (FOS). Journal of Management, 36,1372-1405.

[55] Rode, J. C., Judge, T. A., \& Sun, J. (2012). Incremental validity of core self-evaluations in the presence of other self-concept traits: An investigation of applied psychology criteria in the United States and China. Journal of Leadership \& Organizational Studies, 19, 326-340.

[56] Diener, E., Emmons, R. A., Larsen, R. J., \& Griffin, S. (1985). The satisfaction with life scale. Journal of Personality Assessment, 49, 71-75.

[57] Cortina, L. M., Magley, V. J., Williams, J. H., \& Langhout, R. D. (2001). Incivility in the workplace: Incidence and impact. Journal of Occupational Health Psychology, 6, 64-80.

[58] Scott C. Douglas \& Mark J. Martinko. (2001). Exploring the role of individual differences in the prediction of workplace aggression. Journal of Applied Psychology, 86 (4), 547-559.

[59] Mensch, B. S., \& Kandel, D. B. (1998). The effects of performance attributions and outcome dependence on leader feedback behavior following poor subordinate performance. Journal of Organizational Behavior, 19, 259-327.

[60] Diener, E., Louis Tay, Shigehiro Oishi. (2013). Rising income and the Subjective Well-Being of Nations. Journal of Personality and Social Psychology, 104 (2), 267-276.

[61] Buchholz, R. (1978). An empirical study of contemporary beliefs about work in American society. Journal of Applied Psychology, 63, 219-227. Brandt, A. S. (1980). Relationship of locus of control, environmental constraint, length of time in the institution and twenty-one other variables to morale and life satisfaction in the institutionalized elderly (Doctoral dissertation, Texas Woman's University, 1979 ). Dissertation Abstracts International, 40. 5802B. (University Microfilms No. 80-12,153)

[62] Zhang, S \& Wang, L. (2017). Interactional Justice Moderated the Effect of Protestant Work Ethic on Social Loafing. Journal of Advances in Management Sciences \& Information Systems, 2017, 3, 27-35.

[63] Diener, E. (2012). Why societies need happiness and national accounts of well-being. The ranking of happiness in Mexico, $13-31$

[64] Twenge, J. M., Baumeister, R. F., Tice, D. M., \& Stucke, T. S. (2001). If you can't join them, beat them: Effects of social exclusion on aggressive behavior. Journal of Personality and Social Psychology, 81, 1058-1069.

[65] Boehm, J. K., \& Lyubomirsky, S. (2008). Does happiness lead to career success? Journal of Career Assessment, 16, 101-116.

[66] Dunn, J. R., \& Schweitzer, M. E. (2004). Invidious comparisons and insidious behaviors: Envy and social undermining at work. Working paper.

[67] Zhang, S., Liu, W., \& Liu, X. (2012). Investigating the relationship between Protestant Work Ethic and Confucian Dynamism: an empirical test in mainland China. Journal of Business Ethics, 106, 243-252.

[68] Shin, N., Vaughn, B. E., Akers, V., Kim, M., Stevens, S., Krzysik, L., Korth, B. (2011). Are happy children socially successful? Testing a central premise of positive psychology in a sample of preschool children. Journal of Positive Psychology, 6, 355-367.

[69] Andersson, L., \& Pearson, C. (1999). Tit for tat? The spiraling effect of incivility in the workplace. Academy of Management Review, 24, 452-471.

[70] Leung, A. S. M., Wu, L., Chen, Y., \& Young, M. N. (2011). The impact of Workplace Ostracism in service organizations. International Journal of Hospitality Management, 30, 836-844.

[71] Conna Yang. (2014). Does ethical leadership lead to happy workers? A study on the impact of ethical leadership, Subjective Well-Being, and life happiness in the Chinese culture. Journal of Business Ethics, 123, 513-525.

[72] Cook, T. D., \& Campbell, D. T. (1979). Quasi experimentation: Design and analytical issues for field settings. Chicago, IL: Rand McNally.

[73] Hui, C. H. (1988). Measurement of individualism-collectivism. Journal of Research in Personality, 22, 17-36.

[74] Wilkins, A., \& Dyer, W. Jr. (1988). Toward culturally sensitive theories of cultural change. Academy of Management Review, 13 (4), $522-533$.

[75] Weber, M. (1905). The Protestant Ethic and the Spirit of Capitalism, Scribner, New York. 